\title{
A influência do jateamento na cimentação de bandas ortodônticas*
}

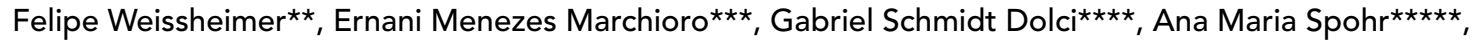

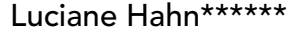

\section{Resumo}

Objetivo: comparar a resistência ao cisalhamento de cinco materiais utilizados na cimentação de bandas ortodônticas: um cimento de ionômero de vidro convencional (Ketac Cem), dois cimentos de ionômero de vidro modificados por resina (3M Multicure Glass Ionomer Cement e Fuji Ortho LC) e duas resinas compostas modificadas por poliácidos (Ultra Band-Lok e Transbond Plus). Metodologia: sobre a superfície de um segmento de banda (6 x $6 \mathrm{~mm}$ ) foi posicionada uma matriz com um orifício de $3 \mathrm{~mm}$ de diâmetro na qual foi inserido o material para cimentação. Metade da amostra foi jateada com partículas de óxido de alumínio. Os corpos-de-prova foram armazenados a $37^{\circ} \mathrm{C}$ por 24 horas em umidade relativa de $100 \% \mathrm{e}$ submetidos ao teste de cisalhamento a uma velocidade de $0,5 \mathrm{~mm} / \mathrm{min}$. Utilizou-se ANOVA e o teste de comparações múltiplas de Tukey para comparar os grupos em cada tipo de superfície estudada (com ou sem jateamento), e o teste $t$ Student para amostras pareadas para a comparação dos grupos jateados e não-jateados $(\mathrm{p}=0,01)$. Resultados: nos grupos não-jateados, Ultra Band-Lok apresentou uma resistência de união significativamente maior $(p<0,01)$ que a obtida pelos outros materiais, os quais não apresentaram diferença significante entre si. Após o jateamento, as resinas compostas modificadas por poliácidos apresentaram uma resistência de união significativamente maior $(\mathrm{p}<0,01)$ que a obtida pelos demais cimentos, sendo que $3 \mathrm{M}$ Multicure Glass Ionomer apresentou um valor de união superior $(\mathrm{p}<0,01)$ ao obtido pelo Ketac Cem e Fuji Ortho LC. Conclusão: o jateamento aumentou significativamente $(p<0,01)$ a resistência de união em todos os grupos.

Palavras-chave: Cimentação de bandas. Jateamento. Resistência ao cisalhamento.

* Resumo da Dissertação apresentada como parte dos requisitos necessários para a obtenção do título de Mestre em Odontologia, área de concentração Ortodontia e Ortopedia Facial, pela Pontifícia Universidade Católica do Rio Grande do Sul.

** Professor de Ortodontia da ULBRA-Torres, Mestre em Ortodontia e Ortopedia Facial pela PUCRS, Especialista em Ortodontia e Ortopedia Facial pela UFPR.

*** Professor de Ortodontia da PUCRS, Doutor em Ortodontia e Ortopedia Facial pela UNESP-Araraquara, Mestre em Ortodontia e Ortopedia Facial pela UFRJ.

**** Mestre em Ortodontia e Ortopedia Facial pela PUCRS.

$\star \star \star \star \star$ Professora de Materiais Dentários da PUCRS, Doutora em Materiais Dentários pela UNICAMP.

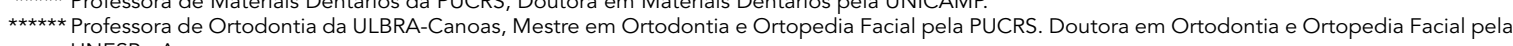
UNESP - Araraquara. 


\section{INTRODUÇÃO}

A cimentação de bandas nos dentes posteriores é um procedimento bastante comum na prática ortodôntica, visto que a colagem de acessórios nesses dentes apresenta um maior índice de falhas ${ }^{8,16,18,22}$. Uma união estável entre o dente e a banda é fundamental para a correta aplicação de forças e conseqüentemente para o sucesso do tratamento ortodôntico. Vários tipos de cimentos têm sido utilizados na cimentação de bandas ortodônticas e a contínua evolução na busca de novos materiais tem contribuído para o aprimoramento da prática clínica.

Alguns estudos demonstraram que as falhas de união entre o dente e a banda ocorrem quase invariavelmente na interface cimento-ban-

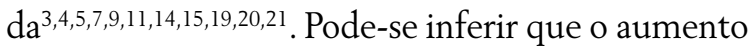
da adesão nessa interface poderia diminuir a ocorrência de falhas.

Considerando-se que o jateamento da superfície interna de bandas ortodônticas com óxido de alumínio é um procedimento que aumenta consideravelmente a resistência de união entre o dente e a banda $1,9,10,13,14,21,23$, este estudo teve por objetivo comparar a resistência ao cisalhamento de um cimento de ionômero de vidro convencional, dois cimentos de ionômero de vidro modificados por resina e duas resinas compostas modificadas por

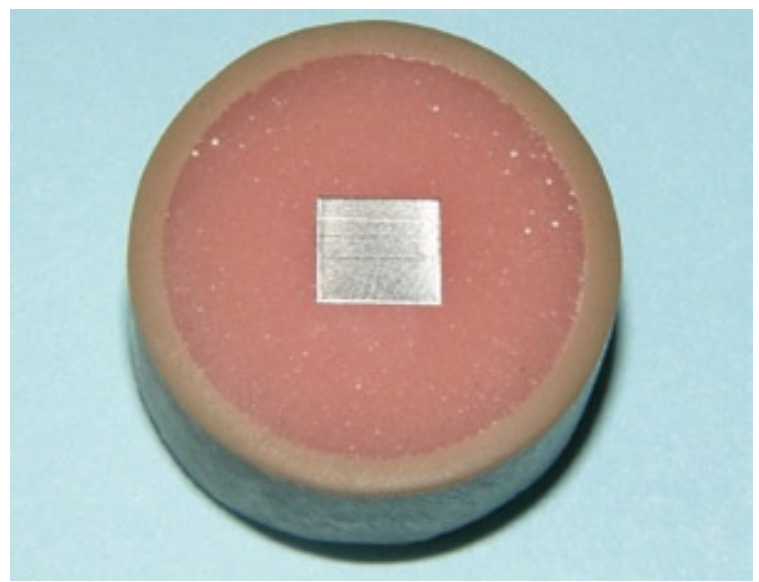

FIGURA 1 - Segmento de banda ortodôntica colado em resina acrílica incluída em tubo de PVC. poliácidos quando aplicados sobre duas superfícies, sem e com jateamento, assim como determinar a influência do jateamento na resistência de união dos materiais analisados.

\section{MATERIAL E MÉTODOS}

Para a confecção da amostra foram utilizados 150 tubos de PVC com $25 \mathrm{~mm}$ de diâmetro e $20 \mathrm{~mm}$ de altura, os quais foram preenchidos com resina acrílica autopolimerizável. Em uma de suas extremidades (Fig. 1) foi colado um segmento de fita de aço inoxidável $(6 \mathrm{~mm} \times 6 \mathrm{~mm})$ utilizado para confecção de bandas ortodônticas (American Orthodontics, EUA). Metade da amostra teve a superfície do segmento de banda jateada por partículas de óxido de alumínio com $50 \mu \mathrm{m}$ de diâmetro (BioArt, Brasil). O jateamento foi realizado durante 6 segundos utilizando-se um aparelho (Micro-etcher, Danville Engineering Inc., EUA) mantendo uma distância padrão de $10 \mathrm{~mm}$ entre a ponta do aparelho e a superfície a ser jateada.

Diferentes tipos de materiais ionoméricos foram utilizados para a confecção dos corpos-deprova (Quadro 1). A amostra $(n=150)$ foi dividida em 10 grupos $(n=15)$, de acordo com o material utilizado para a confecção dos corpos-de-prova e o tratamento aplicado sobre a superfície do segmento de banda (Quadro 2). Os corpos-de-prova fo-

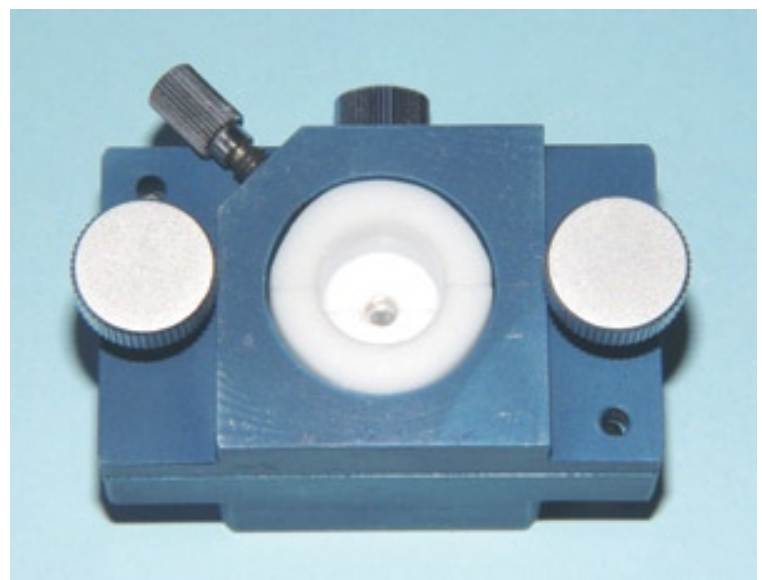

FIGURA 2 - Dispositivo utilizado para a confecção dos corpos-de-prova. 
ram confeccionados utilizando-se um dispositivo constituído por dois suportes metálicos e uma matriz bipartida de Teflon ${ }^{\circledR}$ que apresentava em sua porção interna um orifício de $3 \mathrm{~mm}$ de diâmetro por $2 \mathrm{~mm}$ de altura, no qual foi inserido o material para cimentação (Fig. 2).

Os materiais foram manipulados de acordo com as recomendações do fabricante. Para a inserção do cimento de ionômero de vidro convencional (Ketac Cem) e dos cimentos de ionômero de vidro modificados por resina (3M Multicure Glass

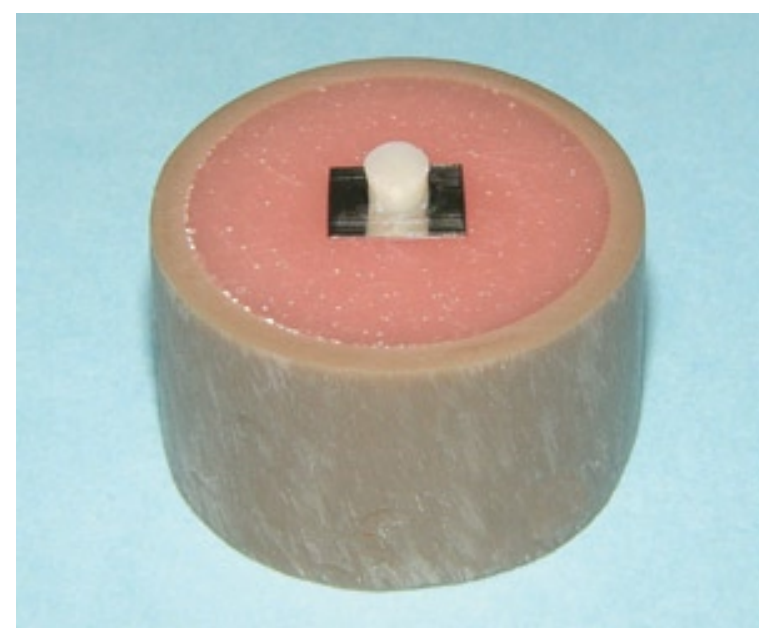

FIGURA 3 - Corpo-de-prova.

\begin{tabular}{|c|c|c|}
\hline Material & Descrição & Reação de Presa \\
\hline Ketac Cem & $\begin{array}{l}\text { Cimento de ionômero } \\
\text { de vidro convencional }\end{array}$ & $\begin{array}{l}\text { Reação ácido- } \\
\text { base }\end{array}$ \\
\hline $\begin{array}{l}\text { 3M Multicure Glass } \\
\text { Ionomer Cement }\end{array}$ & $\begin{array}{l}\text { Cimento de ionômero } \\
\text { de vidro modificado } \\
\text { por resina }\end{array}$ & $\begin{array}{l}\text { Reação ácido- } \\
\text { base, fotoativação } \\
\text { e polimerização } \\
\text { química }\end{array}$ \\
\hline Fuji Ortho LC & $\begin{array}{l}\text { Cimento de ionômero } \\
\text { de vidro modificado } \\
\text { por resina }\end{array}$ & $\begin{array}{l}\text { Reação ácido- } \\
\text { base, fotoativação } \\
\text { e polimerização } \\
\text { química }\end{array}$ \\
\hline Ultra Band-Lok & $\begin{array}{l}\text { Resina composta } \\
\text { modificada por } \\
\text { poliácidos }\end{array}$ & Fotoativação \\
\hline Transbond Plus & $\begin{array}{l}\text { Resina composta } \\
\text { modificada por } \\
\text { poliácidos }\end{array}$ & Fotoativação \\
\hline
\end{tabular}

Quadro 1 - Materiais utilizados para a confecção dos corpos-de-prova.
Ionomer e Fuji Ortho LC), foi utilizado uma seringa Centrix. Para a inserção das resinas compostas modificadas por poliácidos (Ultra Band-Lok e Transbond Plus), foi empregada uma espátula metálica. No grupo Ketac Cem, aguardou-se por 10 minutos a reação de presa inicial do material antes da remoção do dispositivo de fixação. Nos demais grupos os materiais foram fotopolimerizados durante 40 segundos. Após a polimerização, o dispositivo metálico foi desmontado e a matriz bipartida de Teflon ${ }^{\circledR}$ foi removida, visualizando-se um cilindro constituído pelo material ionomérico (Fig. 3). Imediatamente após a sua confecção, os corpos-de-prova foram armazenados em estufa para cultura a $37^{\circ} \mathrm{C}$ com umidade relativa de $100 \%$ por 24 horas.

Procedeu-se então ao teste de resistência ao cisalhamento em máquina de ensaio universal. Os corpos-de-prova foram montados em um dispositivo cilíndrico metálico com diâmetro interno de $25 \mathrm{~mm}$ fixado à máquina de ensaio. A tensão de cisalhamento foi aplicada com auxílio de um fio ortodôntico de $0,45 \mathrm{~mm}$ de diâmetro posicionado na base do cilindro com direção de tração perpendicular ao mesmo, a uma velocidade de $0,5 \mathrm{~mm} /$ minuto, até ocorrer a ruptura da união (Fig. 4).

\begin{tabular}{ccc}
\hline Material & $\mathbf{n}$ & Tratamento \\
\hline Ketac Cem & 15 & Sem jateamento \\
3M Multicure Glass & 15 & Com jateamento \\
lonomer & 15 & Sem jateamento \\
Fuji Ortho LC & 15 & Com jateamento \\
& 15 & Sem jateamento \\
Ultra Band-Lok & 15 & Com jateamento \\
& 15 & Sem jateamento \\
Transbond Plus & 15 & Com jateamento \\
& 15 & Sem jateamento \\
\hline
\end{tabular}

Quadro 2 - Divisão por grupos de acordo com o material utilizado para a confecção dos corpos-de-prova e o tratamento aplicado sobre a superfície do segmento de banda. 


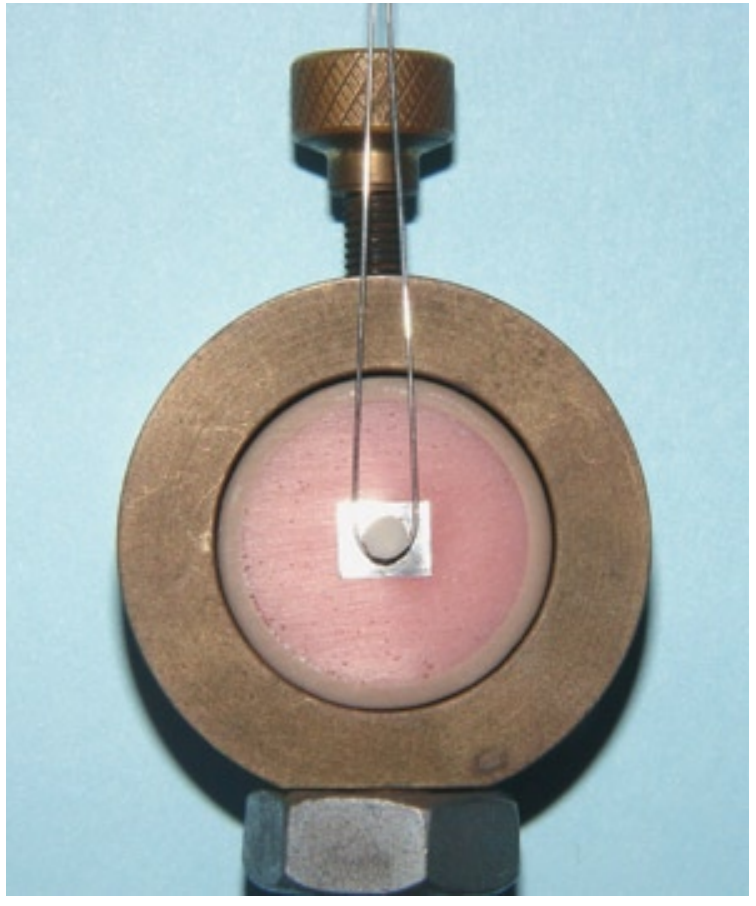

FIGURA 4 - Tensão de cisalhamento aplicada com auxílio de um fio ortodôntico.

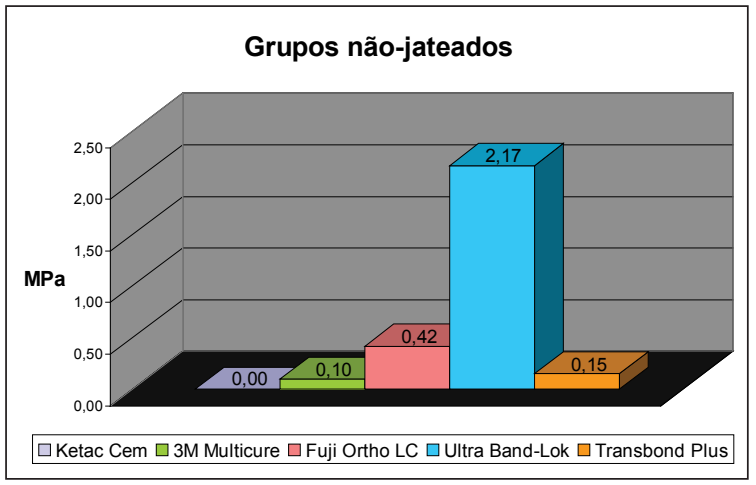

GRÁFICO 1 - Comparação entre os grupos não-jateados.

Tabela 1 - Resultados da comparação entre os grupos nãojateados.

\begin{tabular}{ccccc}
\hline Grupo & $\mathbf{n}$ & $\begin{array}{c}\text { Média } \\
\text { (MPa) }\end{array}$ & $\begin{array}{c}\text { Desvio-padrão } \\
\text { (MPa) }\end{array}$ & p \\
\hline Ketac Cem & 8 & $0,00^{\mathrm{B}}$ & 0,00 & $<0,01^{*}$ \\
3M Multicure & 15 & $0,10^{\mathrm{B}}$ & 0,04 & \\
Fuji Ortho LC & 13 & $0,42^{\mathrm{B}}$ & 0,29 & \\
Ultra Band-Lok & 15 & $2,17^{\mathrm{A}}$ & 1,04 & \\
Transbond Plus & 15 & $0,15^{\mathrm{B}}$ & 0,03 & \\
\hline
\end{tabular}

* Médias seguidas de mesma letra não diferem significativamente entre si.
Com o objetivo de comparar os grupos entre si para cada tipo de superfície estudada (não-jateada ou jateada) foi realizado o teste estatístico Análise de Variância (ANOVA) e o teste de comparações múltiplas de Tukey ao nível de significância de $1 \%$. Para a comparação dos grupos correspondentes a um mesmo material com diferentes tipos de superfície (não-jateada e jateada) foi empregado o teste estatístico de comparações de médias $t$ Student para amostras pareadas ao nível de significância de $1 \%$.

\section{RESULTADOS}

Na comparação entre os grupos não-jateados, o maior valor médio de união foi obtido pelo Ultra Band-Lok $(2,17 \pm 1,04 \mathrm{MPa})$, seguido pelo Fuji Ortho LC $(0,42 \pm 0,29 \mathrm{MPa})$, Transbond Plus $(0,15 \pm 0,03 \mathrm{MPa}), 3 \mathrm{M}$ Multicure Glass Ionomer $(0,10 \pm 0,04 \mathrm{MPa})$ e Ketac Cem (0,00 0 0,00MPa). Pelos resultados do teste estatístico Análise de Variância (ANOVA) e do teste de comparações múltiplas de Tukey, verificou-se a existência de diferença significante entre os grupos estudados $(\mathrm{p}<0,01)$. Observou-se que o grupo Ultra Band-Lok apresentou uma média significativamente superior à dos outros grupos, os quais não apresentaram diferença significante entre si (Tab. 1, Gráf. 1).

Entre os grupos jateados, o maior valor médio de união foi obtido pelo Transbond Plus $(9,99 \pm 2,27 \mathrm{MPa})$, seguido pelo Ultra Band-Lok $(9,33 \pm 1,62 \mathrm{MPa}), 3 \mathrm{M}$ Multicure Glass Ionomer $(4,38 \pm 0,98 \mathrm{MPa})$, Ketac Cem $(2,35 \pm 1,04 \mathrm{MPa})$ e Fuji Ortho LC $(2,16 \pm 1,12 \mathrm{MPa})$. Pelos resultados do teste estatístico Análise de Variância (ANOVA) e do teste de comparações múltiplas de Tukey, verificou-se a existência de diferença significante entre os grupos estudados $(\mathrm{p}<0,01)$. Observou-se que os grupos Transbond Plus e Ultra Band-Lok apresentaram uma média significativamente superior à média dos outros grupos, seguidos pelo grupo 3M Multicure (que difere de todos) e, por último, com menores médias, pelos grupos Ketac Cem e Fuji Ortho LC, os quais não diferiram entre si (Tab. 2, Gráf. 2). 
Tabela 2 - Resultados da comparação entre os grupos jateados.

\begin{tabular}{ccccc}
\hline Grupo & n & $\begin{array}{c}\text { Média } \\
\text { (MPa) }\end{array}$ & $\begin{array}{c}\text { Desvio-padrão } \\
\text { (MPa) }\end{array}$ & p \\
\hline Ketac Cem & 15 & $2,35^{\mathrm{C}}$ & 1,04 & $<0,01^{*}$ \\
3M Multicure & 15 & $4,38^{\mathrm{B}}$ & 0,98 & \\
Fuji Ortho LC & 15 & $2,16^{\mathrm{C}}$ & 1,12 & \\
Ultra Band-Lok & 15 & $9,33^{\mathrm{A}}$ & 1,62 & \\
Transbond Plus & 15 & 9,99 & 2,27 & \\
\hline
\end{tabular}

* Médias seguidas de mesma letra não diferem significativamente entre si.

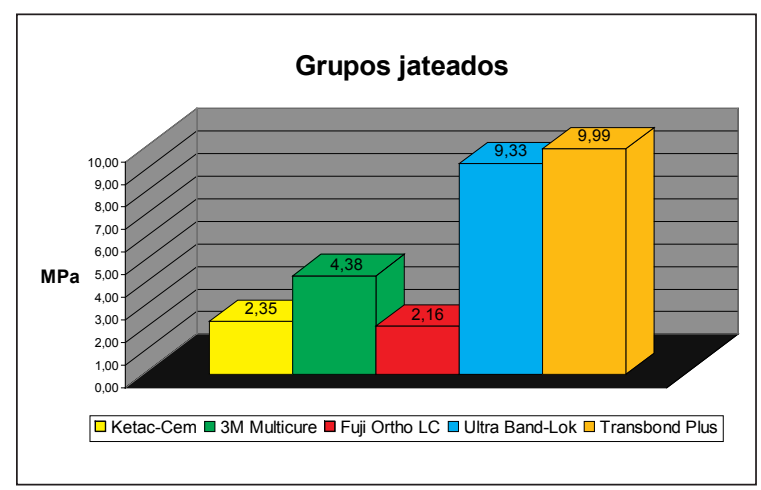

GRÁFICO 2 - Comparação entre os grupos jateados.

Os resultados do teste de comparações de médias $t$ Student para amostras pareadas indicaram uma diferença significante entre as superfícies jateada e não-jateada para todos os grupos estuda$\operatorname{dos}(\mathrm{p}<0,01)$. Observou-se que as médias foram superiores nas superfícies em que foi realizado o jateamento (Tab. 3).

\section{DISCUSSÃO}

Neste estudo a resina composta modificada por poliácidos Ultra Band-Lok (2,17MPa) apresentou uma resistência de união significativamente maior $(\mathrm{p}<0,01)$ que os demais materiais quando aplicada sobre a superfície não-jateada. Com exceção desse material, a união química e/ou mecânica dos materiais analisados sobre a superfície da banda sem jateamento foi bastante tênue. A maior resistência ao cisalhamento do Ultra Band-Lok pode ser atribuída a uma maior união química do ma-
Tabela 3 - Resultados da comparação entre os grupos nãojateados e jateados.

\begin{tabular}{|c|c|c|c|c|}
\hline Grupo & n & $\begin{array}{l}\text { Média } \\
\text { (MPa) }\end{array}$ & $\begin{array}{l}\text { Desvio-pa- } \\
\text { drão (MPa) }\end{array}$ & $\mathbf{p}$ \\
\hline \multicolumn{5}{|l|}{ KETAC CEM } \\
\hline $\begin{array}{c}\text { sem } \\
\text { jateamento }\end{array}$ & 8 & 0,00 & 0,00 & $<0,01$ \\
\hline $\begin{array}{c}\text { com } \\
\text { jateamento }\end{array}$ & 8 & 2,71 & 1,18 & \\
\hline \multicolumn{5}{|c|}{ 3M MULTICURE } \\
\hline $\begin{array}{c}\text { sem } \\
\text { jateamento }\end{array}$ & 15 & 0,10 & 0,04 & $<0,01$ \\
\hline $\begin{array}{c}\text { com } \\
\text { jateamento }\end{array}$ & 15 & 4,38 & 0,98 & \\
\hline \multicolumn{5}{|c|}{ FUJI ORTHO LC } \\
\hline $\begin{array}{c}\text { sem } \\
\text { jateamento }\end{array}$ & 13 & 0,42 & 0,29 & $<0,01$ \\
\hline $\begin{array}{c}\text { com } \\
\text { jateamento }\end{array}$ & 13 & 2,18 & 1,15 & \\
\hline \multicolumn{5}{|l|}{$\begin{array}{c}\text { ULTRA } \\
\text { BAND-LOK }\end{array}$} \\
\hline $\begin{array}{c}\text { sem } \\
\text { jateamento }\end{array}$ & 15 & 2,17 & 1,04 & $<0,01$ \\
\hline $\begin{array}{c}\text { com } \\
\text { jateamento }\end{array}$ & 15 & 9,33 & 1,62 & \\
\hline \multicolumn{5}{|l|}{$\begin{array}{l}\text { TRANSBOND } \\
\text { PLUS }\end{array}$} \\
\hline $\begin{array}{c}\text { sem } \\
\text { jateamento }\end{array}$ & 15 & 0,15 & 0,03 & $<0,01$ \\
\hline $\begin{array}{c}\text { com } \\
\text { jateamento }\end{array}$ & 15 & 9,99 & 2,27 & \\
\hline
\end{tabular}

terial à superfície metálica da banda e/ou a uma maior união micromecânica. A retenção mecânica é apontada por alguns autores como o principal fator responsável pela união entre o cimento e o metal após a cimentação de bandas ${ }^{5,17,23}$. No microscópio eletrônico de varredura, pode-se observar que a superfície da banda, aparentemente lisa, possui inúmeras irregularidades que podem auxiliar na retenção do material. A capacidade do Ultra Band-Lok de penetrar nessas irregularidades pode ser maior que aquela apresentada pelos outros materiais. De acordo com Millett, Kamahli e $\mathrm{McColl}^{15}$, o aumento da viscosidade favorece a resistência de união, e o Ultra Band-Lok é o material que apresenta o maior grau de viscosidade. 
Segundo Anusavice ${ }^{2}$, em uma união mecânica a força de retenção depende da resistência do cimento, portanto uma maior resistência coesiva do Ultra Band-Lok poderia explicar o seu melhor desempenho em relação aos outros materiais.

$\mathrm{O}$ jateamento aumentou significativamente $(\mathrm{p}<0,01)$ a resistência de união em todos os grupos analisados. Esse aumento pode ser devido a uma melhora na união química entre a superfície metálica da banda e o material ou a uma retenção micromecânica mais eficiente ${ }^{23}$. De acordo com alguns autores, a remoção parcial da camada de óxidos encontrada na superfície da banda pelo jateamento favorece a união química entre o cimento e o metal ${ }^{12,23}$. Esse fato poderia explicar o aumento na adesão entre os diferentes materiais avaliados e a banda após o jateamento. No entanto, a resistência de união da maioria dos grupos não-jateados é bastante fraca. Pode-se supor que a união química entre a superfície metálica da banda e o cimento não exerce um papel preponderante na união entre essas duas superfícies. Um aumento da união micromecânica explicaria de forma mais consistente as modificações ocorridas nos valores de união após o jateamento. De acordo com Wood, Paleczny e Johnson ${ }^{23}$, a união mecânica entre o cimento e o metal é o principal determinante na resistência de união de bandas jateadas. Após o jateamento, verificouse um aumento significativo das zonas retentivas na mesma superfície, além de uma maior área de união, o que favorece a união entre o cimento e o metal ${ }^{14,23,24}$.

No presente estudo, a realização do jateamento produziu um aumento significativo $(\mathrm{p}<0,01)$ da resistência de união em todos os grupos estudados. Portanto pode-se deduzir que o jateamento da superfície interna de bandas ortodônticas com partículas de óxido de alumínio tende a diminuir a ocorrência de falhas de união. Este estudo procurou auxiliar o profissional na sua prática clínica, fornecendo evidências que possam diminuir o insucesso na cimentação de bandas.

\section{CONCLUSÕES}

A resina composta modificada por poliácidos Ultra Band-Lok apresentou uma resistência de união significativamente maior que a obtida pelos outros materiais, os quais não apresentaram diferença significante entre si, quando aplicada sobre um segmento de banda não-jateado;

Após o jateamento, as resinas compostas modificadas por poliácidos Ultra Band-Lok e Transbond Plus apresentaram uma resistência de união significativamente maior que a obtida pelos outros materiais, sendo que o cimento de ionômero de vidro modificado por resina 3M Multicure Glass Ionomer apresentou um valor de união superior ao obtido pelo cimento de ionômero de vidro convencional Ketac Cem e pelo cimento de ionômero de vidro modificado por resina Fuji Ortho LC;

$\mathrm{O}$ jateamento aumentou significativamente a resistência de união em todos os grupos analisados. 


\title{
The effect of sandblasting in band cementation
}

\begin{abstract}
Aim: to compare the shear-peel bond strength of five orthodontic cements: a conventional glass ionomer cement (Ketac Cem), two resin-modified glass ionomer cements (3M Multicure Glass lonomer and Fuji Ortho LC) and two polyacid-modified composite resin cement (Ultra Band-Lok and Transbond Plus). Methods: cements were placed in a $3 \mathrm{~mm}$ diameter mold at bonding interface and bonded to $6 \times 6 \mathrm{~mm}$ standard and sandblasted stainless steel band specimens mounted to acrylic blocks. Specimens were stored at $37^{\circ} \mathrm{C}$ and $100 \%$ relative humidity for 24 hours and debonded in tension on a testing machine at $0.05 \mathrm{~cm} /$ minutes. Data were analyzed by one-way analysis of variance (ANOVA) followed by a Tukey multiple comparison procedure in the same surface treatment groups (sandblasted or non-sandblasted) and by paired t-tests in different surface treatment groups (sandblasted and non-sandblasted) $(p=0.01)$. Results: Ultra Band-Lok demonstrated significant greater shear-peel bond strength $(p<0.01)$ in the non-sandblasted groups, and no statistically significant differences were noted between others cements. In the sandblasted groups, resin-modified glass ionomer cements demonstrated the highest mean bond strength $(p<0.01)$, and 3M Multicure Glass lonomer showed a greater shear-peel bond strength $(p<0.01)$ than Ketac Cem and Fuji Ortho LC. Conclusion: sandblasting increased shear-peel bond strength in all tested groups $(p<0.01)$.
\end{abstract}

Key words: Orthodontic banding. Sandblasting. Shear-peel bond strength.

\section{REFERÊNCIAS}

1. ABU KASIM, N. K; MILLETT, D. T.; McCABE, J. F. The ball mill as a means of investigating the mechanical failure of dental material. J Dent, Bristol, v. 24, no. 1/2, p.117-124, Jan./ Mar. 1996.

2. ANUSAVICE, K. J. Phillips materiais dentários. 10. ed. Rio de Janeiro: Guanabara Koogan, 1998.

3. FRICKER, J. P.; MCLACHLAN, M. D. Clinical studies of glass ionomer cements Part 1 - A twelve month clinical study comparing zinc phosphate cement to glass ionomer. Aust Orthod J, Brisbane, v. 9, no. 1, p. 179-180, mar. 1985.

4. FRICKER, J. P.; MCLACHLAN, M. D. Clinical studies of glass ionomer cements. Part 2 - A two year clinical study comparing glass ionomer cement with zinc phosphate cement. Aust Orthod J, Brisbane, v. 10, no. 1, p. 12-14, mar. 1987

5. FRICKER, J. P. A 12-month clinical comparison of resin-modified light-activated adhesives for the cementation of orthodontic molar bands. Am J Orthod Dentofacial Orthop, St. Louis, v. 112 , no. 3, p. 239-243, sept. 1997.

6. GILLGRASS, T. J. et al. Fluoride release, microbial inhibition and microleakage pattern of two orthodontic band cements. J Dent Bristol, v. 27, no. 6, p. 455-461, aug. 1999

7. GILLGRASS, T. J. et al. Modified composite or conventional glass ionomer for band cementation? A comparative clinical trial. Am J Orthod Dentofacial Orthop, St. Louis, v. 120, no. 1, p. 49-53, july 2001.

8. GOTTLIEB, E. L.; NELSON, A. H.; VOGELS, D. S. $1986 \mathrm{JCO}$ study of orthodontic diagnosis and treatment procedures. J Clin Orthod, Boulder, v. 20, no. 9, p. 612-625, sept. 1986

9. GÜRAY, E.; KARAMAN, A. l. Effects of different roughening methods on band retention. J Clin Orthod, Boulder, v. 31, no. 6, p. 361-366, june 1997.

10. HODGES, S. J.; GILTHORPE, M. S.; HUNT, N. P. The effect of micro-etching on the retention of orthodontic molar bands: a clinical trial. Eur J Orthod, Oxford, v. 23, no. 1, p. 91-97, feb. 2001.

11. MAIJER, R.; SMITH, D.C. A comparison between zinc phosphate and glass ionomer cement in Orthodontics. Am J Orthod Dentofacial Orthop, St. Louis, v. 93, no. 4, p. 273-279, apr. 1988.

12. McCONNELL, R. J. Metal-resin bonding. J Calif Dent Assoc, San Francisco, v. 21, no. 6, p. 38-42, june 1993.

13. MENNEMEYER, V. A.; NEUMAN, P.; POWERS, J. M. Bonding of hybrid ionomers and resin cements to modified orthodontic band materials. Am J Orthod Dentofacial Orthop, St. Louis, v. 115 , no. 2, p. 143-147, feb. 1999.

14. MILLETT, D. T. et al. The effect of sandblasting on the retention of first molar orthodontic bands cemented with glass ionomer cement. Br J Orthod, Oxford, v. 22, no. 2, p. 161-169, may 1995.

15. MILLETT, D. T.; KAMAHLI, K.; McCOLL, J. Comparative laboratory investigation of dual-cured vs. conventional glass ionomer cements for band cementation. Angle Orthod, Appleton, v. 68, no. 4, p. 345-350, aug. 1998.

16. MILLETT, D. T. et al. Bonded molar tubes: a retrospective evaluation of clinical performance. Am J Orthod Dentofacial Orthop, St. Louis, v. 115, no. 6, p. 667-674, june 1999.

17. MILLETT, D. T. et al. A clinical retrospective evaluation of 2 orthodontic band cements. Angle Orthod, Appleton, v. 71, no. 6, p. 470-476, dec. 2001

18. MIZRAHI, E. Orthodontic bands and directly bonded brackets: a review of clinical failure rate. J Dent, Bristol, v. 11, no. 3, p. 231-236, sept. 1983.

19. MIZRAHI, E. Glass ionomer cements in orthodontics: an update. Am J Orthod Dentofacial Orthop, St. Louis, v. 93, no. 6, p. 505-507, june 1988

20. NORRIS, D. S. et al. Retention of orthodontic bands with new fluoride-releasing cements. Am J Orthod Dentofacial Orthop, St. Louis, v. 89, no. 3, p. 206-211, mar. 1986.

21. SEEHOLZER, H. W.; DASCH, W. Banding with glass ionomer cement. J Clin Orthod, Boulder, v. 22, no. 3, p. 165-169, mar. 1988.

22. STIRRUPS, D. R. A comparative clinical trial of a glass ionomer and a zinc phosphate cement for securing orthodontic bandas. Br J Orthod, Oxford, v. 18, no. 1, p.15-20, feb. 1991.

23. WOOD, D. P.; PALECZNY, G. J.; JOHNSON, L. N. The effect of sandblasting on the retention of orthodontic bands. Angle Orthod, Appleton, v. 66, no. 3, p. 207-214, june 1996.

24. ZACHRISSON, B. U.; BUYUKYILMAZ, T. Recent advances in bonding to gold, amalgam and porcelain. J Clin Orthod, Boulder, v. 27 , no. 12, p. $661-675$, dec. 1993.

\section{Endereço de correspondência}

Felipe Weissheimer

Av. Carlos Gomes, 141/809 - Porto Alegre/RS

CEP: 90.480-003

E-mail: felweiss@terra.com.br 